\title{
On Computational Modelling of Strain-Hardening Material Dynamics
}

\author{
Philip Barton ${ }^{1, *}$ and Evgeniy Romenski ${ }^{2}$ \\ 1 Graduate Aerospace Laboratories, California Institute of Technology, California \\ 91125, USA. \\ ${ }^{2}$ Sobolev Institute of Mathematics, Russian Academy of Sciences, Novosibirsk 630090, \\ Russia.
}

Received 17 December 2010; Accepted (in revised version) 27 May 2011

Available online 23 December 2011

\begin{abstract}
In this paper we show that entropy can be used within a functional for the stress relaxation time of solid materials to parametrise finite viscoplastic strainhardening deformations. Through doing so the classical empirical recovery of a suitable irreversible scalar measure of work-hardening from the three-dimensional state parameters is avoided. The success of the proposed approach centres on determination of a rate-independent relation between plastic strain and entropy, which is found to be suitably simplistic such to not add any significant complexity to the final model. The result is sufficiently general to be used in combination with existing constitutive models for inelastic deformations parametrised by one-dimensional plastic strain provided the constitutive models are thermodynamically consistent. Here a model for the tangential stress relaxation time based upon established dislocation mechanics theory is calibrated for OFHC copper and subsequently integrated within a two-dimensional moving-mesh scheme. We address some of the numerical challenges that are faced in order to ensure successful implementation of the proposed model within a hydrocode. The approach is demonstrated through simulations of flyer-plate and cylinder impacts.
\end{abstract}

PACS: 81.40.Ef, 83.85.St, 83.60.La, 81.40.Lm, 83.10.Gr

Key words: Entropy, viscoplasticity, solid mechanics, relaxation time, Maxwell solid, Eulerian.

\section{Introduction}

It is widely recognised that physically consistent continuum models for governing strainhardening inelastic deformations in solid materials require knowledge of certain internal variables. The irreversibility of these processes reflects the need for a suitable irreversible

*Corresponding author. Email addresses: ptbarton@caltech.edu, phil.t.barton@gmail.com (P. Barton), evrom@math.nsc.ru (E. Romenski) 
measure within the material. For calculations of uniaxial loading tests, formulation of the constitutive models for the flow stress, or stress rate for viscoplastic models, in terms of the easily determinable plastic strain is adequate for this purpose. Ordinarily the principle of such calculations is to calibrate the constitutive models for inelastic deformations, through comparison of the theory with available experimental data, with the eventual intent of integrating these within a more complex model for simulations in higher space dimensions for which the uniaxial model is a subset. However, employing the plasticity models within a multi-dimensional framework is complicated since the strain then forms a potentially non-spherical rank-two tensor. Assuming the material of interest is isotropic, the frame indifferent plasticity models derived in this way employ only scalar measures of state which must thus be recovered in some way through functionals of the higher-dimensional state variables. The classical Lagrangian models that include the process of work hardening have relied upon a one-dimensional measure of plastic strain determined through integration of the equivalent plastic strain rate to acquire the Von Mises strain. Within Eulerian formulations the permissible advection of material through control volumes requires the strain history to be tracked, and the Von Mises plastic strain is typically declared as an internal parameter for which an additional transport law can be derived with the production defined on the basis of, for example, the Levy-St. Venant flow rule. In both the Lagrangian and Eulerian variants of the continuum theory the determination and use of equivalent plastic strain in models describing inelastic deformations is empirical. This paper is devoted to investigating the axiom that the thermodynamically consistent entropy can instead be used as the internal variable parametrising strain hardening phenomena.

In the absence of external sources, the net increase in entropy of a system can be attributed to increasing internal disorder. It is mentioned that in [4] entropy was successfully used in place of an additional empirical history parameter to describe damage mechanics. For solid materials subjected to loads which result in inelastic deformations it is well known that the observed macroscopic behaviours for strain-hardening materials relate to changes in the microstructure; specifically changes in the number density and motion of dislocations. It is natural to assume then that since changes in the dislocation structure in a solid will contribute to the entropy production, this entropy can be used in a continuum model to parametrise the work-hardening behaviour. As a preliminary, incorporation of the microscopic inelastic dynamics within a continuum framework is possible by using statistical plasticity based upon ensemble averages of the dislocation kinetics in the construction of constitutive models for inelastic deformations. Thus constitutive models employ only continuum parameters such as invariants of stress and onedimensional plastic strain, yet reflect microscopic changes in response to macroscopic disturbances. Much of the dislocation theory is now well established and is attributed to the early efforts of Orowan [28], Taylor [32] and Gillis and Gilman [9-12]. It is this physically consistent theory that forms the basis of successful continuum plasticity models [35]. It remains to quantify some function of the entropy to substitute plastic strain in parametrising the strain-hardening behaviour through changes in the mobile dislocation 
density.

In the subsequent sections Eulerian constitutive laws shall be presented for elastoplastic solid materials, and the related closure models derived. The Eulerian framework is considered since it is within this setting that use of an entropy based model for inelastic deformations is expected to yield the greatest benefits for the aforementioned reasons. Specifically we shall focus on a model for solid dynamics in which the thermodynamically compatible balance laws are expressible as a first order system of partial differential equations in divergent form $[15,30]$. For compressible problems such models are becoming increasingly popular (see for example $[1-3,6,8,17,20,25,26,33]$ ) over classical empirical variants based upon advection of the deviatoric stresses since numerical methods can be applied that circumvent the requirement to explicitly include artificial viscosity for resolving shocks, thus improving the accuracy of calculations. The proposed functional for the Maxwell relaxation time of tangential stress, governing the limit of allowable elastic deformations due to plastic yield, is derived based upon dislocation kinetics and calibrated for OFHC copper using available experimental data for uniaxial loading tests. Finally, the performance of the complete model is analysed by comparing computations with experimental data for a range of cylinder impact tests.

\section{Governing constitutive models}

The state of solid media is assumed to be entirely parametrised by the velocity components $u_{i}$, elastic deformation gradients $F_{i j}^{e}$ and entropy $\mathscr{S}$. It is thus possible [15] to formulate the constitutive equations governing the dynamics of solid materials in the Eulerian frame of reference as a first order system in conservative form. In Cartesian coordinates $x_{k}$ the system comprises conservation laws for momentum, deformation, and energy:

$$
\begin{aligned}
& \frac{\partial \rho u_{i}}{\partial t}+\frac{\partial\left(\rho u_{i} u_{k}-\sigma_{i k}\right)}{\partial x_{k}}=0, \\
& \frac{\partial \rho F_{i j}^{e}}{\partial t}+\frac{\partial\left(\rho F_{i j}^{e} u_{k}-u_{i} \rho F_{k j}^{e}\right)}{\partial x_{k}}=-u_{i} \beta_{j}+\rho \varphi_{i j}, \\
& \frac{\partial \rho\left(\mathscr{E}+u_{i} u_{i} / 2\right)}{\partial t}+\frac{\partial\left(\rho u_{k}\left(\mathscr{E}+u_{i} u_{i} / 2\right)-u_{i} \sigma_{i k}\right)}{\partial x_{k}}=0,
\end{aligned}
$$

where repeated indices denote summation. The specific internal energy is a function of the state variables:

$$
\mathscr{E}=\mathscr{E}\left(\mathbf{F}^{e}, \mathscr{S}\right),
$$

and the described materials are assumed to be hyperelastic such that the Cauchy stresses are a function of the thermodynamic derivatives:

$$
\sigma_{i j}=\rho F_{i k}^{e} \frac{\partial \mathscr{E}}{\partial F_{j k}^{e}}
$$


Density is related to the deformation gradients, $\rho=\rho_{0} / \operatorname{det}\left|\mathbf{F}^{e}\right|$, through the reference value $\rho_{0}$ (taken to correspond to the unstressed state). The vector $\beta_{j}:=\partial \rho F_{k j}^{e} / \partial x_{k}$ on the right-hand side of Eq. (2.1) is introduced to ensure the conservative form of the equations for $\mathbf{F}^{e}=\left[F_{i j}^{e}\right]$ and can be treated as a source term [30]. The mass continuity equation does not appear explicitly in Eq. (2.1), although the system is fully determined. It is noted however that this is satisfied through the equations for $\mathbf{F}^{e}$. It remains to specify the form of the source $\varphi_{i j}$ restricting permissible values of $F^{e}$ in the event of inelastic deformations.

Note that the spectral decomposition of the elastic deformation gradient tensor can be written:

$$
\mathbf{F}^{e}=\mathbf{U K}^{e} \mathbf{V},
$$

such that $\mathbf{U}, \mathbf{V}$ are orthogonal rotation matrices, and $\mathrm{K}^{e}=\operatorname{diag}\left(k_{1}^{e}, k_{2}^{e}, k_{3}^{e}\right)$ the diagonal matrix of principle elastic stretches $k_{i}^{e}, i=1,2,3$ (eigenvalues). Various elastic strain tensors can be determined from the rotation and stretch matrices (see [15]). In what follows we shall make use of the Henky (logarithmic) strain tensor:

$$
\mathbf{H}:=\mathbf{U}\left(\ln \mathbf{K}^{e}\right) \mathbf{U}^{\mathrm{T}},
$$

where $\mathbf{U}^{\mathrm{T}}$ is the transpose of $\mathbf{U}$.

The tensor $\varphi_{i j}$ is chosen such that the material deforms in way phenomenologically analogous to the Maxwell solid model. Deviatoric stresses are thus limited via a relaxation time $\tau$ (proportional to the reciprocal of Maxwell viscosity) which can be a function of the state variables:

$$
\tau=\tau\left(\mathbf{F}^{e}, \mathscr{S}\right)
$$

For finite deformations the source can be defined in different manners and we take it in the form

$$
\varphi:=-\frac{1}{\tau} \mathbf{H}^{\prime} \mathbf{F}^{e} .
$$

where $\mathbf{H}^{\prime}:=\mathbf{H}-(\operatorname{Tr} \mathbf{H}) \mathbf{I} / 3$ denotes the deviator of the matrix $\mathbf{H}$.

The conservative system can be shown to permit the following non-conservative evolution equation for entropy:

$$
\frac{\partial \mathscr{S}}{\partial t}+u_{k} \frac{\partial \mathscr{S}}{\partial x_{k}}=\frac{1}{T}\left(\frac{\partial \mathscr{E}\left(\mathbf{F}^{e}, \mathscr{S}\right)}{\partial \mathbf{F}^{e}}\right): \varphi,
$$

where the temperature is found from the thermodynamic derivative of internal energy density: $T=\partial \mathscr{E} / \partial \mathscr{S}$. Note that Eq. (2.7) is strictly valid only for smooth flows, since in according to Eq. (2.7), in the special case that all total deformations remain elastic $\left(\varphi_{i j}=0\right)$ the entropy remains equal to zero if zero initially. However, an additional entropy growth appears across shocks, which are defined as discontinuous solutions of the system (2.1) through the Rankine-Hugoniot conditions. Thus it can be assumed that the solutions of Eq. (2.7) in non-smooth flows gives values of entropy specifically associated with plastic 
work. To formalise this point we recast Eq. (2.7) introducing the plastic entropy as an additional internal state variable $\mathscr{S}^{p}$ :

$$
\frac{\partial \mathscr{S}^{p}}{\partial t}+u_{k} \frac{\partial \mathscr{S}^{p}}{\partial x_{k}}=\frac{1}{T(\rho, \mathscr{S})}\left(\frac{\partial \mathscr{E}\left(\mathbf{F}^{e}, \mathscr{S}\right)}{\partial \mathbf{F}^{e}}\right): \varphi\left(\mathbf{F}^{e}, \mathscr{S}, \mathscr{S}^{p}\right),
$$

and distinguish that $\mathscr{S}^{p}$ in smooth elastoplastic flows is equivalent to the total entropy $\mathscr{S}$, and otherwise for flows with shocks it is the entropy due to plastic work. Thus the following inequality should always be satisfied:

$$
\mathscr{S}^{p} \leq \mathscr{S}
$$

Thus, for shocked flows the complete system for dynamics of elastoplastic materials with hardening consists of the system (2.1) and Eq. (2.8) for plastic entropy.

It is straightforward to show that for the particular choice of $\varphi$, for $\tau>0$ and $T>0$ the entropy production is positive if

$$
\begin{aligned}
\left(k_{1}^{e} \mathscr{E}_{k_{1}^{e}}-k_{2}^{e} \mathscr{E}_{k_{2}^{e}}\right)\left(\ln k_{1}^{e}-\ln k_{2}^{e}\right) & +\left(k_{2}^{e} \mathscr{E}_{k_{2}^{e}}^{e}-k_{3}^{e} \mathscr{E}_{k_{3}^{e}}^{e}\right)\left(\ln k_{2}^{e}-\ln k_{3}^{e}\right) \\
& +\left(k_{3}^{e} \mathscr{E}_{\frac{k_{3}^{e}}{e}}-k_{1}^{e} \mathscr{E}_{k_{1}^{e}}\right)\left(\ln k_{3}^{e}-\ln k_{1}^{e}\right) \geq 0
\end{aligned}
$$

Hence the formulation satisfies the Clausius-Duhem inequality for an adiabatically isolated system provided the function for specific internal energy satisfies Eq. (2.10). We can be assured then that this is the case provided the specific internal energy is chosen in a physically consistent way; that is it reflects what is seen experimentally. It remains to specify the closing relations for the specific internal energy and relaxation time of tangential stresses.

\section{Closure models}

The first of the closure models we consider is the relaxation time of tangential stress. Following [22], the relaxation time is assumed to be linearly inversely proportional to the one-dimensional measure of plastic strain rate:

$$
\tau \sim a / \dot{\epsilon}_{p},
$$

where $a$ is the constant of proportionality. For a physically consistent description of the dependence of $\dot{\epsilon}_{p}$ we take the dislocation kinetic description of Orowan [28] which for strain hardening materials and one-dimensional states of stress and strain can be written:

$$
\dot{\epsilon}_{p}=|\mathbf{b}| N\left(\epsilon_{p}\right) f\left(\epsilon_{p}\right) v\left(\epsilon_{p}, \sigma_{t}\right)
$$

where $\mathbf{b}$ is the Burgers vector, $N$ is the density of mobile dislocations, $0<f\left(\epsilon_{p}\right) \leq 1$ is the fraction of total dislocations that are mobile which provides the mechanism for strainhardening, $v$ the velocity of these dislocations, and $\sigma_{t}$ a one-dimensional measure of the 
tangential stress. We assume the following forms for the functionals, the first two of which are widely accepted:

$$
N=\left(N_{0}+M \epsilon_{p}\right), \quad v=v_{0} \exp \left(-\frac{D}{\sigma_{t}}\right), \quad f=\exp \left(-\frac{H \epsilon_{p}{ }^{n}}{\sigma_{t}}\right),
$$

where $N_{0}$ is the initial density, $M$ a multiplication parameter, $D$ is the characteristic drag stress, $v_{0}$ the limiting velocity which can sensibly be taken to equal to the reference speed of shear waves $b_{0}$. It is mentioned that although the form for $v$ might not adequately describe the dislocation motion of all solid materials [13], it is desirable to develop a model that when integrated within a multi-dimensional framework is guaranteed not to exceed a saturation level in the event of over-stress. In the model for $f$, taken from [12], the exponent can be varied arbitrarily to accommodate the desired non-linearity, but here we restrict the range to $0 \leq n \leq 1$ to conform with the requirement that the deformations asymptote to a maximum in the limit of finite strains $\epsilon_{p} \rightarrow \infty$. Note that an alternative approach to introducing a mechanism for strain hardening proposed in [12] is to take $f=1$ and modify the characteristic drag stress to be some function of the plastic strain such that the dislocation velocity increases with increasing plastic strain. This approach is taken in [24] where it is suggested that non-linear hardening can be accounted for by assuming the drag stress is a polynomial of higher order: $D=\mathcal{P}\left(\epsilon_{p}\right)$. In fact both interpretations are equivalent and choosing either makes no difference to the final form of Eq. (3.2).

Eqs. (3.2)-(3.3), provide an analytic expression for the relaxation time. We now require a function for the plastic strain in terms of the entropy to complete the model.

To prove that entropy can instead be used as a parameter for describing work hardening we consider the special case of uniaxial loading of a thin cylindrical bar. We are further motivated to develop this theory to enable the subsequent calibration of material dependent parameters entering into our model, through comparison of solutions with experimental data for uniaxial tension/compression bar experiments. We consider a uniform bar orientated along the $x_{1}$-direction subjected to a one dimensional velocity field thus resulting in a constant longitudinal strain rate $\dot{\epsilon}$. The conditions result in a state of triaxial deformation, $\mathbf{F}^{e}=\mathbf{K}^{e}$ with $k_{2}^{e}=k_{3}^{e}$, since $\mathbf{U}=\mathbf{V}=\mathbf{I}$; and uniaxial stress, $\sigma=\operatorname{diag}\left(\sigma_{1}, \sigma_{2}, \sigma_{3}\right)$ with $\sigma_{2}=\sigma_{3}=0$. Introducing the substantial derivative

$$
\frac{\mathrm{d}}{\mathrm{d} t}=\frac{\partial}{\partial t}+u_{k} \frac{\partial}{\partial x_{k}}
$$

determination of the homogeneous state of the bar $\left(k_{1}^{e}, k_{2}^{e}, \mathscr{S}\right)$ at any point in time thus reduces to solving:

$$
\frac{\mathrm{d} k_{1}^{e}}{\mathrm{~d} t}=\dot{\epsilon} k_{1}^{e}-\frac{2 k_{1}^{e}}{3 \tau\left(k_{1}^{e}, k_{2}^{e}, \mathscr{S}\right)} \ln \frac{k_{1}^{e}}{k_{2}^{e}}, \quad \frac{\mathrm{d} \mathscr{S}}{\mathrm{d} t}=\frac{2 k_{1}^{e} \mathscr{E}_{1}^{e}}{3 T \tau\left(k_{1}^{e}, k_{2}^{e}, \mathscr{S}\right)} \ln \frac{k_{1}^{e}}{k_{2}^{e}}
$$




$$
\begin{aligned}
& \sigma_{1}=\left.\frac{\rho_{0}}{k_{2}^{e^{2}}} \mathscr{E}_{k_{1}^{e}}^{e}\left(k_{1}^{e}, k_{2}^{e}, k_{3}^{e}, \mathscr{S}\right)\right|_{k_{3}^{e}=k_{2}^{e}} \\
& \sigma_{2}=\sigma_{3}=\left.\frac{\rho_{0}}{k_{1}^{e} k_{2}^{e}} \mathscr{E}_{k_{2}^{e}}\left(k_{1}^{e}, k_{2}^{e}, k_{3}^{e}, \mathscr{S}\right)\right|_{k_{3}^{e}=k_{2}^{e}}=0,
\end{aligned}
$$

closed by the choice of energy density function and relaxation time. Note that here we consider smooth solutions for which, as was noted in Section 2, entropy and plastic entropy coincide and here we do not make a distinction between them. To further develop our model, however, it is convenient to reformulate the entropy law in terms of the plastic work rate.

Denote by $h_{i}:=\ln k_{i}$ the principle Henky strain components, and consider the additive decomposition into elastic and plastic parts:

$$
h_{i}=h_{i}^{e}+h_{i}^{p},
$$

where $h_{i}^{e}:=\ln k_{i}^{e}$ and $h_{i}^{p}:=\ln k_{i}^{p}$ are the elastic and plastic Henky strains respectively. Using the fact that $\dot{k}_{1}=k_{1} \dot{h}_{1}$, and $\dot{\epsilon}=\mathrm{d} h_{1} / \mathrm{d} t$ in the equation for $k_{1}^{e}$ in (3.5) gives

$$
\frac{\mathrm{d} h_{1}^{p}}{\mathrm{~d} t}=\frac{2}{3} \frac{1}{\tau} \ln \left(\frac{k_{1}^{e}}{k_{2}^{e}}\right)
$$

Using this result the entropy production rate can be written

$$
\frac{\mathrm{d} \mathscr{S}}{\mathrm{d} t}=\frac{\sigma_{1}}{\rho T} \frac{\mathrm{d} h_{1}^{p}}{\mathrm{~d} t} .
$$

which assuming that the density variation is small gives

$$
\rho_{0} \int_{\mathscr{S}_{0}}^{\mathscr{S}} T \mathrm{~d} \mathscr{S}=\int_{0}^{\epsilon_{p}} \sigma_{1} \mathrm{~d} \epsilon_{p}
$$

where $\epsilon_{p}=h_{1}^{p}$ is the plastic deformation. Suppose that during plastic deformation the stress $\sigma_{1}\left(\dot{\epsilon}_{1}\right) \geq \sigma_{y}\left(\dot{\epsilon}_{1}\right)$, where $\sigma_{y}\left(\dot{\epsilon}_{1}\right)$ denotes the initial rate-dependent flow stress, can be represented as a function of the plastic strain $\sigma_{1}=\sigma_{1}\left(\dot{\epsilon}_{1}, \epsilon_{1}^{p}\right)$. Then Eq. (3.9) can be used to formulate entropy as a function of plastic strain provided the temperature and stress are known.

Consider first determination of the temperature through specification of the internal energy functional. The specific internal energy can be decomposed into potentials describing the cold compression, $\mathscr{E}_{c}(\rho, \mathscr{S})$, thermal energy density, $\mathscr{E}_{t}(\rho, \mathscr{S})$, and the contribution due to shear strain, $\mathscr{E}_{S}\left(\mathbf{F}^{e}, \mathscr{S}\right)$. A more general function might also include a contribution due to changes in dislocation density, $\mathscr{E}_{h}\left(\mathbf{F}^{e}, \mathscr{S}\right)$ [31]. Thus, a general form could be written

$$
\mathscr{E}\left(\mathbf{F}^{e}, \mathscr{S}\right)=\mathscr{E}_{c}(\rho, \mathscr{S})+\mathscr{E}_{t}(\rho, \mathscr{S})+\mathscr{E}_{s}\left(\mathbf{F}^{e}, \mathscr{S}\right)+\mathscr{E}_{h}\left(\mathbf{F}^{e}, \mathscr{S}\right)
$$


Within the cold compressional and elastic energies, the entropy appears through the potential temperature dependence of the bulk and shear elastic moduli respectively. For the contribution due to hardening the energy can be expressed as a function of the dislocation density and thus the entropy dependency is unambiguous as a result of the axiom for which this paper is devoted. However, the term should also contain a modulus which, like the previously mentioned elastic moduli, could be a function of temperature. Based on Eq. (3.10) the total temperature is:

$$
T\left(\mathbf{F}^{e}, \mathscr{S}\right)=T_{c}(\rho, \mathscr{S})+T_{t}(\rho, \mathscr{S})+T_{s}\left(\mathbf{F}^{e}, \mathscr{S}\right)+T_{h}\left(\mathbf{F}^{e}, \mathscr{S}\right),
$$

where the subscripts denote the derivatives with respect to entropy of the different energies: $T_{c}:=\partial \mathscr{E}_{c} / \partial \mathscr{S}, T_{t}:=\partial \mathscr{E}_{t} / \partial \mathscr{S}, T_{s}:=\partial \mathscr{E}_{s} / \partial \mathscr{S}, T_{h}:=\partial \mathscr{E}_{h} / \partial \mathscr{S}$. In [31] it is shown that, at least for a number of common metals, that $T_{t} \gg T_{c}+T_{s}+T_{h}$, hence $T \sim T_{t}$. This result is assumed to hold in our analysis. Assuming that density variations are small, the entropy change can be given by the classical Mie-Grüneisen form [34] for the special case of isovolumetric heating, which rearranges to give:

$$
T=T_{0} \exp \left(\frac{\mathscr{S}-\mathscr{S}_{0}}{C_{\mathrm{V}}}\right)
$$

where $\mathscr{S}_{0}$ is the entropy at the reference temperature $T_{0}$, and $C_{\mathrm{v}}$ is the heat capacity at constant volume. Substituting this result in Eq. (3.9) relates the entropy distribution as a function of plastic strain:

$$
\rho_{0} T_{0} C_{\mathrm{V}}\left[\exp \left(\frac{\mathscr{S}-\mathscr{S}_{0}}{C_{\mathrm{V}}}\right)-1\right]=\int_{0}^{\epsilon_{p}} \sigma_{1} \mathrm{~d} \epsilon_{p}
$$

Fig. 1(a) shows the entropy production computed using Eq. (3.13) for the experimentally determined stress-strain data extracted from [16]; the result is of course rate-dependent. For use in the model for relaxation time the function relating entropy and plastic strain must be independent of rate-effects. To assist in deriving this desired result it is constructive to first explore certain empirical relations for the stress as a function of plastic strain. In what follows we shall make reference to the plastic work defined as

$$
W:=\int_{0}^{\epsilon_{p}} \sigma_{1} \mathrm{~d} \epsilon_{p} .
$$

Consider Ludwik's [21] relation for the flow stress $\sigma_{1}=\sigma_{y}+K(\dot{\epsilon}) \epsilon_{p}{ }^{n}$, where $\sigma_{y}$ is the initial flow stress, $K$ is the rate dependent strength index, and $n$ the work hardening exponent. If $\sigma_{y}=0$ then the plastic work is $W=K \epsilon_{p}{ }^{n+1} /(n+1)$, which when divided through by the stress gives a parameter

$$
\Phi:=W / \sigma_{1}
$$




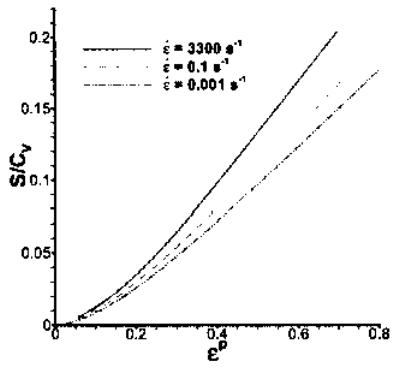

(a)

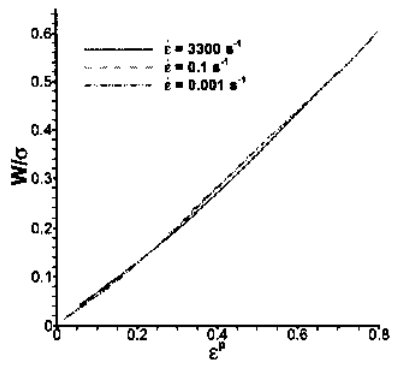

(b)

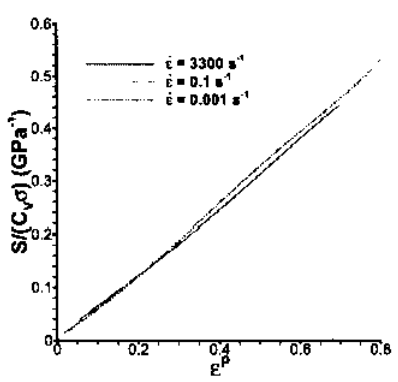

(c)

Figure 1: Profiles of (a) non-dimensionalised entropy calculated using Eq. (3.13) against plastic strain; (b) ratio of plastic work to stress against plastic strain; (c) non-dimensionalised entropy over stress against plastic strain, for the experimental stress-strain data extracted from [16].

which is not only independent of strain but is also linear: $\Phi=\epsilon^{p} /(n+1)$. For the more realistic case of $\sigma_{y} \neq 0$ rate effects are not entirely eliminated in the non-dimensional parameter $\Phi$, however it is found that these terms are small and the function $\Phi\left(\epsilon_{p}\right)$ is approximately linear to a high degree of accuracy for a number of common metals. These are of course only empirical results, but we find that the same is found for the experiment (Fig. 1(b)). It is with confidence then that we continue with assumption that $\Phi\left(\epsilon_{p}\right)$ is linear. We further postulate that the non-dimensionalised entropy $\mathscr{S} / C_{\mathrm{V}}$ is small such that for the case of zero initial entropy the rate-independent entropy distribution is given by

$$
\frac{\left(\mathscr{S} / C_{\mathrm{v}}\right)}{\sigma_{1}} \sim \frac{1}{\rho_{0} T_{0} C_{\mathrm{v}}} \Phi .
$$

Based upon these results, and the fact that it can be shown for the experimental data that Eq. (3.16) can be approximated well by a linear relation between $\mathscr{S} / C_{\mathrm{V}}$ and $\epsilon_{p}$ (Fig. 1(c)), we propose that

$$
\epsilon_{p} \sim C_{\epsilon_{p}}\left(\frac{\left(\mathscr{S} / C_{\mathrm{V}}\right)}{\sigma_{1}}\right)
$$

where $C_{\varepsilon_{p}}$ is a constant to be determined.

Recalling that we supposed in this Section that $\mathscr{S}=\mathscr{S}^{p}$, the final model for the relaxation time Eqs. (3.2)-(3.3) thus becomes

$$
\tau=\tau_{0}\left[N_{0}+M\left(C_{\epsilon_{p}} \frac{\mathscr{S}^{p}}{C_{\mathrm{v}} \sigma_{1}}\right)\right]^{-1} \exp \left\{\frac{1}{\sigma_{1}}\left[D+H\left(C_{\epsilon_{p}} \frac{\mathscr{S}^{p}}{C_{\mathrm{V}} \sigma_{1}}\right)^{n}\right]\right\}
$$

where the constant of proportionality $a$, magnitude of Burgers vector, and shear wave speed $b_{0}$ have been consolidated into a single material constant $\tau_{0}=a /\left(|\mathbf{b}| b_{0}\right)$ for clarity. To generalise this result to three-dimensional stress states we replace $\sigma_{1}$ with the invariant measure of tangential stress intensity $\sigma_{e q}:=\sqrt{\left(2 \sigma_{i j}^{\prime} \sigma_{i j}^{\prime}\right) / 3}$. 
It remains to specify analytic forms for the internal energies appearing in Eq. (3.10). For an isotropic hyperelastic material the internal energy density can be formulated in terms of the invariants of any chosen strain tensor, $\mathcal{I}_{p}$, with $p=1,2,3: \mathscr{E}=\mathscr{E}\left(\mathcal{I}_{1}, \mathcal{I}_{2}, \mathcal{I}_{3}, \mathscr{S}\right)$. Semi-empirical formulations are taken for each contribution [5]:

$$
\begin{aligned}
& \mathscr{E}_{C}\left(\mathcal{I}_{3}, \mathscr{S}\right)=\frac{K_{0}}{2 \alpha^{2}}\left(\mathcal{I}_{3}^{\alpha / 2}-1\right)^{2}, \\
& \mathscr{E}_{t}\left(\mathcal{I}_{3}, \mathscr{S}\right)=C_{\mathrm{v}} T_{0} \mathcal{I}_{3}^{\gamma / 2}\left[\exp \left(\frac{\mathscr{S}-\mathscr{S}_{0}}{C_{\mathrm{v}}}\right)-1\right], \\
& \mathscr{E}_{S}\left(\mathcal{I}_{1}, \mathcal{I}_{2}, \mathcal{I}_{3}\right)=\frac{B_{0}}{2} \mathcal{I}_{3}^{\beta / 2}\left(\mathcal{I}_{1}^{2} / 3-\mathcal{I}_{2}\right),
\end{aligned}
$$

where the invariants

$$
\mathcal{I}_{1}:=\operatorname{Tr}(\mathbf{G}), \quad \mathcal{I}_{2}:=\frac{1}{2}\left[(\operatorname{Tr}(\mathbf{G}))^{2}-\operatorname{Tr}\left(\mathbf{G}^{2}\right)\right], \quad \mathcal{I}_{3}:=\operatorname{det}|\mathbf{G}|=\left(\rho / \rho_{0}\right)^{2},
$$

correspond to those of the elastic Finger tensor $G:=F^{e^{-T}} F^{e^{-1}}$. Note that we have neglected the contribution due to strain-hardening. The parameters $K_{0}:=c_{0}^{2}-(4 / 3) b_{0}^{2}, B_{0}:=b_{0}^{2}$ are the squared bulk speed of sound and the squared reference speed of transverse shear waves, respectively; $\alpha, \beta, \gamma$ are constants characterising the nonlinear dependence of the sound speeds and temperature on the compression.

\section{Determination of parameters for OFHC Copper}

Only a portion of the material parameters entering in to the proposed model for relaxation time Eq. (3.18) have been reported in the open literature for a wide range of materials of interest. Thus, those that remain outstanding must be determined through some other means. We follow the commonly employed technique of modifying the unknown parameters to provide a best fit of numerically obtained data for uniaxial loading of a thin bar to that from experiments of equivalent systems. Specifically, given a set of $N_{\mathrm{S}}$ data points from experimental stress-strain curves $\sigma_{\mathrm{EXP}}(\dot{\epsilon}, \epsilon)$, for $N_{\mathrm{SR}}$ different strain rates, we attempt to minimise the sum of squared residuals:

$$
\mathscr{R}\left(c_{1}, c_{2}, \cdots, c_{n}\right)=\sum_{i=1}^{N_{\mathrm{SR}}} \sum_{j=1}^{N_{\mathrm{S}}}\left(\sigma_{\mathrm{EXP}}\left(\dot{\epsilon}_{i}, \epsilon_{j}\right)-\sigma_{\mathrm{NUM}}\left(\dot{\epsilon}_{i}, \epsilon_{j}, c_{1}, c_{2}, \cdots, c_{n}\right)\right)^{2}
$$

for the $n$ number of outstanding material parameters $c_{i}, i=1, \cdots, n$, where $\sigma_{\mathrm{NUM}}$ is the stress determined through solution of the constitutive equations (3.5) with Eq. (3.18) and Eq. (3.19).

The material of interest here is annealed oxygen-free high conductivity copper (OFHC $\mathrm{Cu}$ ) for which experimental deformation curves are presented in [16] for the reference temperature $T_{0}$ and different strain-rates. The elastic constants used in the equation of 
Table 1: Material parameters for the internal energy equation-of-state for OFHC Cu.

\begin{tabular}{||lll||}
\hline Parameter & Value & Units \\
\hline$\rho_{0}$ & 8.93 & $\mathrm{~g} \cdot \mathrm{cm}^{-3}$ \\
$c_{0}$ & 4.6 & $\mathrm{~km} \cdot \mathrm{s}^{-1}$ \\
$c_{\mathrm{V}}$ & $3.9 \cdot 10^{-4}$ & $\mathrm{~kJ} \cdot \mathrm{g}^{-1} \cdot \mathrm{K}^{-1}$ \\
$T_{0}$ & 300 & $\mathrm{~K}$ \\
$b_{0}$ & 2.1 & $\mathrm{~km} \cdot \mathrm{s}^{-1}$ \\
$\alpha$ & 1.0 & - \\
$\beta$ & 3.0 & - \\
$\gamma$ & 2.0 & - \\
\hline
\end{tabular}

Table 2: Material parameters for the relaxation time model calibrated for OFHC Cu.

\begin{tabular}{||lll||}
\hline Parameter & Value & Units \\
\hline$\tau_{0}$ & $8.5 \cdot 10^{-6}$ & $\mu \mathrm{s} \cdot \mathrm{cm}^{-2}$ \\
$N_{0}$ & $10^{9}$ & $\mathrm{~cm}^{-2}$ \\
$M$ & $10^{11}$ & $\mathrm{~cm}^{-2}$ \\
$D$ & 0.79 & $\mathrm{GPa}$ \\
$H$ & 21.1 & $\mathrm{GPa}$ \\
$C_{\epsilon_{p}}$ & 1.38 & - \\
$n$ & 0.49 & - \\
\hline
\end{tabular}

state for specific energy Eq. (3.19) are presented in Table 1. For the relaxation model, values for the initial dislocation density and multiplication coefficient, $N_{0}$ and $M$, are readily available in the open literature [19] and thus remained fixed during the model calibration. The constant of proportionality between the entropy function and plastic strain, $C_{\epsilon_{p}}$ in Eq. (3.17), was predetermined from the experimental data. Hence, solution of Eq. (4.1) is required for $\tau_{0}, D, H$, and $n$. To minimise the multi-parametric function $\mathrm{Eq}$. (4.1) we use the downhill simplex method of Nelder \& Mead [27]. The downhill simplex method is probably the simplest multi-dimensional minimisation method if not the most efficient. On account of numerical stiffness encountered when integrating the system of differential equations for the model due to potentially small values of the relaxation time, the implicit Newton-Raphson method is employed.

Material constants for the relaxation model calibrated for OFHC $\mathrm{Cu}$ are presented in Table 2. The constitutive model was found to adequately describe the deformation curves in comparison to the experiment for the range of strains and strain-rates considered (Fig. 2). The choice of a power law to modify the mobile dislocation fraction proved not to simultaneously accurately represent the small and large strain hardening behaviours and the calibration method is seen to tend toward a compromise of these two regions. However, the gradient of the curves at large strains remains greater than that of the experiment. It is mentioned that if the small strain behaviours are insignificant in comparison to finite deformations, then it might be beneficial to recalibrate the model to reflect this by placing greater weight on minimising residuals at higher strains. 


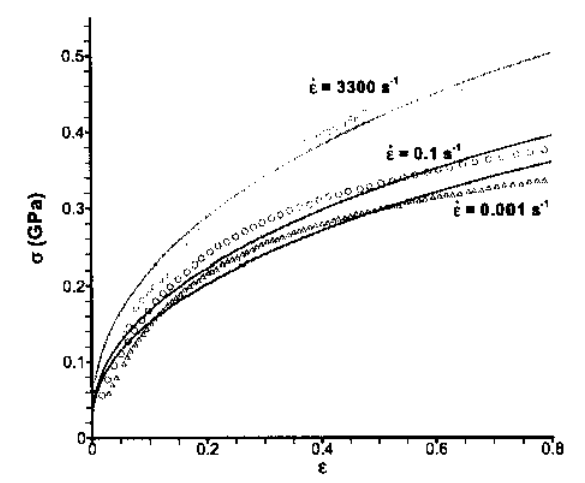

Figure 2: Stress-strain data for the indicated strain rates $(\dot{\epsilon})$ : data computed from the bar theory (lines) in comparison to the experimental data from [16] (symbols). The indicated total strain is $\epsilon=\dot{\epsilon} t$.

\section{Numerical simulations}

In order to examine the capabilities of the proposed model for the relaxation time certain fundamental testcases can be singled out. Specifically the wave properties shall be studied using a flyer plate testcase, and then the ability to resolve strain-hardening inelastic deformations shall be examined using a series of cylinder impact tests. In all examples that follow the material is OFHC Cu with values of the material parameters equal to those reported in the previous section. For the cylinder impact it is necessary to solve the additional geometric source terms in the governing PDEs for cylindrical flows, and to resolve the time-dependent free surfaces and apply the appropriate stress free boundary conditions. Details of the numerical scheme used to resolve the more complex multi-dimensional moving boundary problems shall first be presented. Note that for the one-dimensional examples the following method will simplify significantly.

To enable the resolution of material boundaries a moving mesh scheme is used. The governing constitutive equations are thus recast in a generalised, time-dependent coordinate system $\xi_{i}=\xi_{i}\left(x_{k}, t\right), \tau=\tau(t)$, by using the following identities ( $\tau:=t$ is assumed):

$$
\mathrm{d} x_{k}=\frac{\partial x_{k}}{\partial \xi_{i}} \mathrm{~d} \xi_{i}+\frac{\partial x_{k}}{\partial t} \mathrm{~d} t
$$

After some rearranging the governing equations can be expressed in divergent form:

$$
\begin{aligned}
& \frac{\partial J \rho u_{i}}{\partial t}+\frac{\partial J\left(\rho u_{i} \widehat{u}_{\eta}-\widehat{\sigma}_{i \eta}\right)}{\partial \xi_{\eta}}=-\frac{\phi J}{x_{1}} S_{i}^{m}, \\
& \frac{\partial J \rho F_{i j}^{e}}{\partial t}+\frac{\partial J\left(\rho F_{i j}^{e} \widehat{u}_{\eta}-u_{i} \rho \widehat{F}_{i \eta}^{e}\right)}{\partial \xi_{\eta}}=-J u_{i} \widehat{\beta}_{j}-J \rho \varphi_{i j}-\frac{\phi J}{x_{1}} S_{i j}^{d}, \\
& \frac{\partial J E}{\partial t}+\frac{\partial J\left(\rho \widehat{u}_{\eta} E-u_{i} \widehat{\sigma}_{i \eta}\right)}{\partial \xi_{\eta}}=-\frac{\phi J}{x_{1}} S^{e},
\end{aligned}
$$


with

$$
\widehat{u}_{i}:=\frac{\partial \xi_{i}}{\partial x_{k}}\left(u_{k}-\mu_{k}\right), \quad \widehat{\sigma}_{i j}:=\sigma_{j k} \frac{\partial \xi_{i}}{\partial x_{k}}, \quad \widehat{F}_{j i}^{e}:=F_{k j}^{e} \frac{\partial \xi_{i}}{\partial x_{k}}, \quad \widehat{\beta}_{j}:=\frac{\partial \xi_{\eta}}{\partial x_{k}} \frac{\partial \rho F_{k j}^{e}}{\partial \xi_{\eta}},
$$

where $J:=\left|\partial x_{k} / \partial \xi_{i}\right|$ is the Jacobian determinant; $\mu_{k}:=\partial x_{k} / \partial \tau$ is the grid velocity; $\widehat{\mu}_{i}:=$ $\mu_{k}\left(\partial \xi_{i} / \partial x_{k}\right)$ is the grid velocity vector in curvilinear coordinates. The parameter $\phi$ distinguishes the coordinate system with $\phi=0$ corresponding to Cartesian and $\phi=1$ cylindrical. For the latter the additional geometric source terms, for the special case of zero rotational motion, are:

$$
\begin{aligned}
& \mathbf{S}^{m}=\left(\begin{array}{c}
\rho u_{1}^{2}-\sigma_{11}+\sigma_{33} \\
\rho u_{1} u_{2}-\sigma_{21} \\
0
\end{array}\right), \quad \mathbf{S}^{d}=\rho u_{1}\left(\begin{array}{ccc}
F_{11}^{e} & F_{12}^{e} & F_{13}^{e} \\
F_{21}^{e} & F_{22}^{e} & F_{23}^{e} \\
0 & 0 & 0
\end{array}\right), \\
& S^{e}=\rho u_{1}\left(\mathscr{E}+u_{i} u_{i} / 2\right)-u_{i} \sigma_{1 i},
\end{aligned}
$$

where it is assumed that the axis of symmetry coincides with the $x_{2}$-coordinate axis. The system (5.2) is hyperbolic and has thirteen characteristic waves, which for each coordinate direction $\eta$ have the following speeds: six genuinely non-linear waves with speeds $u_{k} \widetilde{x}_{k}^{\eta} \mathbf{I}-\mathscr{D}^{\eta}$ and $u_{k} \widetilde{x}_{k}^{\eta} \mathbf{I}+\mathscr{D}^{\eta}$, and seven linearly degenerate waves with the coincident speed $u_{k} \widetilde{x}_{k}^{\eta}$. The employed normalised transformation metrics are defined as $\widetilde{x}_{k}^{\eta}:=\left(\partial \zeta^{\eta} / \partial x_{k}\right) / L_{\eta}$ with the arc length $L_{\eta}:=\left|\left(\partial \xi^{\eta} / \partial \mathbf{x}\right)\right|$, whereas the matrix $\mathscr{D}$ is related to the roots of the acoustic tensor:

$$
\Omega_{i j}^{\eta}:=\frac{1}{\rho} \frac{1}{L_{\eta}^{2}} \frac{\partial \hat{\sigma}_{\eta i}}{\partial F_{j k}^{e}} \hat{F}_{k \eta^{\prime}}^{e} \quad k=1,2,3,
$$

(note that $\eta$ denotes the coordinate direction and repetition does not denote summation) which has the spectral decomposition $\Omega=Q^{-1} \mathscr{D}^{2} \mathbf{Q}$.

The equations (5.2) are solved over a computational grid with cells $I_{i, j}$ spanning the material domain in the range $i=1, \cdots, I$ in $\xi_{1}$ and $j=1, \cdots, J$ in $\xi_{2}$. Cell averaged values of the conserved variables at the current time level $t^{n}$ are denoted $(J \rho \mathbf{u})_{i, j}^{n},\left(J \rho \mathbf{F}^{e}\right)_{i, j^{\prime}}^{n}(J \rho E)_{i, j^{\prime}}^{n}$ and stored at the cell centres. The coordinates of cell nodes are also known for the current time-level $x_{i, j}^{n}$ for $i=1, \cdots, I+1, j=1, \cdots, J+1$. The global timestep is chosen on the basis of the discretisation parameters and wavespeeds:

$$
\Delta t=\mathscr{C} \times \min _{i, j}\left(\frac{J}{L_{1} \max (\lambda)_{1}}, \frac{J}{L_{2} \max (\lambda)_{2}}\right),
$$

where $\max (\lambda)_{\eta}$ is the maximum wavespeed in the direction $\eta$, and $0<\mathscr{C} \leq 1 / 2$ is an adjustable scalar parameter used to control the timestep so as to satisfy the CourantFriedrichs-Lewy condition. The time-step chosen on the basis of the wavespeeds may lead to an unstable update of the equations for $\mathrm{F}^{\ell}$ in the event of high strain-rate behaviour since in the limit $\dot{\epsilon} \rightarrow \infty$ then $\tau \rightarrow 0$, and hence $\Delta t / \tau \gg 1$. So as to maintain use 
of discretisation parameters that do not resolve the fine scales of the relaxation operator, but are otherwise adequate, time-operator splitting is used where the convective part of the inhomogeneous system (5.2) is solved initially using an explicit integration method and the solution subsequently used as the initial state for evaluating the relaxation source term $\varphi$ implicitly. To clarify the method we write the system (5.2) in the following matrix form

$$
\frac{\partial \mathbf{U}}{\partial t}+\frac{\partial \mathscr{F} \eta}{\partial \xi_{\eta}}=-\mathbf{S}^{\mathrm{C}}-\mathbf{S}^{\mathrm{G}}-\mathbf{S}^{\mathrm{P}}
$$

where for two spatial dimensions

$$
\begin{aligned}
& \mathbf{U}:=J\left(\begin{array}{c}
\rho \mathbf{u} \\
\rho \mathbf{F}^{\mathrm{T}} \mathbf{e}_{1} \\
\rho \mathbf{F}^{e \mathrm{~T}} \mathbf{e}_{2} \\
\rho E
\end{array}\right), \quad \mathscr{F}:=J\left(\begin{array}{c}
\widehat{u}_{\eta} \rho \mathbf{u}-\widehat{\sigma} \mathbf{e}_{\eta} \\
\widehat{u}_{\eta} \rho \mathbf{F}^{e \mathrm{~T}} \mathbf{e}_{1}-u_{1} \rho \widehat{\mathbf{F}}^{\mathrm{T}} \mathbf{e}_{\eta} \\
\widehat{u}_{\eta} \rho \mathbf{F}^{e \mathrm{~T}} \mathbf{e}_{2}-u_{2} \rho \widehat{\mathbf{F}}^{\mathrm{e}} \mathbf{e}_{\eta} \\
\widehat{u}_{\eta} \rho E-\mathbf{e}_{\eta}^{\mathrm{T}} \cdot(\widehat{\sigma} \mathbf{u})
\end{array}\right), \\
& \mathbf{S}^{\mathrm{C}}:=J\left(\begin{array}{c}
0 \\
u_{1} \widehat{\beta} \\
u_{2} \widehat{\beta} \\
0
\end{array}\right), \quad \mathbf{S}^{\mathrm{G}}:=\frac{\phi J}{x_{1}}\left(\begin{array}{c}
\mathbf{S}^{m} \\
\mathbf{S}^{d^{\mathrm{T}}} \mathbf{e}_{1} \\
\mathbf{S}^{d^{\mathrm{T}}} \mathbf{e}_{2} \\
S^{e}
\end{array}\right), \quad \mathbf{S}^{\mathrm{P}}:=J \rho\left(\begin{array}{c}
0 \\
\varphi^{\mathrm{T}} \mathbf{e}_{1} \\
\varphi^{\mathrm{T}} \mathbf{e}_{2} \\
0
\end{array}\right),
\end{aligned}
$$

and $e_{\eta}$ are the unit vectors. Furthermore, we introduce the operator

$$
\mathbf{L}_{i, j}:=\frac{\mathscr{F}_{i+1 / 2, j}^{1}-\mathscr{F}_{i-1 / 2, j}^{1}}{\Delta \xi_{1}}+\frac{\mathscr{F}_{i, j+1 / 2}^{2}-\mathscr{F}_{i, j-1 / 2}^{2}}{\Delta \xi_{2}}+\mathbf{S}_{i, j}^{\mathrm{C}}+\mathbf{S}_{i, j}^{\mathrm{G}},
$$

which encompasses the discrete representation of the spatial derivatives found using finite differences, and where $\Delta \xi=1$ is assumed. A first order representation of the splitting method can now be written:

$$
\begin{aligned}
& \mathbf{U}_{i, j}^{*}=\mathbf{U}_{i, j}^{n}-\Delta t \mathbf{L}_{i, j}\left(\mathbf{U}^{n}\right), \\
& \mathbf{U}_{i, j}^{n+1}=\mathbf{U}_{i, j}^{*}-\Delta t \mathbf{S}^{\mathrm{P}}\left(\mathbf{U}_{i, j}^{n+1}\right),
\end{aligned}
$$

where $\mathrm{U}^{*}$ denotes the intermediate state. In practice the forward and backward Euler integrations can be replaced by more accurate and stable methods. For the implicit part the backward differentiation formula method in the LSODE solver from Lawrence Livermore National Laboratory [29] is used. For the explicit part the second-order total-variation diminishing Runge-Kutta method is used, where for any vector quantity $\mathscr{Q}$ :

$$
\begin{aligned}
& \mathscr{Q}_{i, j}^{(1)}=\mathscr{Q}_{i, j}^{n}-\frac{1}{2} \Delta t \mathscr{R}_{i, j}\left(\mathscr{Q}^{n}\right), \\
& \mathscr{Q}_{i, j}^{n+1}=\mathscr{Q}_{i, j}^{n}-\frac{1}{2} \Delta t\left[\left(\mathscr{R}_{i, j}\left(\mathscr{Q}^{n}\right)+\mathscr{R}_{i, j}\left(\mathscr{Q}^{(1)}\right)\right],\right.
\end{aligned}
$$

where $\mathscr{R}$ represents the relative right-hand-side terms. For the physical evolution equations $\mathscr{Q}:=\mathbf{U}$ and $\mathscr{R}:=\mathbf{L}$. 
The interior intercell flux functions in Eq. (5.7) are evaluated from solutions of the Riemann problem arising as a result of the jump in cell averaged data at cell boundaries. A linearised Riemann solver is used [1] with increased spatial accuracy achieved through reconstruction of the piecewise constant data using a third-order weighted essentially non-oscillatory (WENO) method [18]. Near boundaries, interfacial fluxes are found through solution of the appropriate multi-component Riemann problem incorporating the relative boundary conditions (e.g. the solid/vacuum Riemann problem at free surfaces enforcing the traction free condition) using the method presented in [3]. The solutions at the grid bounds provide the cell face grid velocities $\mu$ from which the nodal velocities can be found using the arithmetic mean of adjacent faces. Subsequently the boundary nodes can be updated to the next time level by putting $\mathscr{Q}:=\mathrm{x}$ and $\mathscr{R}:=\mu$ in Eq. (5.9). The updated boundary node positions provide the Dirichlet boundary conditions to determine the new positions of the interior nodes, for which a transfinite interpolation method is used. Consequently the interior cell face velocities required in the Riemann solution for all interior cell faces are determined through comparison of the node coordinate values at the old and new time-levels.

\subsection{One-dimensional impact studies}

We first investigate the elastoplastic wave propagation characteristics resulting from a $0.2 \mathrm{~km} \cdot \mathrm{s}^{-1}$ impact of a projectile layer on a target. Both components are assumed to be semi-infinite. Computed profiles of normal velocity and equivalent stress are shown in Fig. 3 for different time instants. One can observe the well known characteristic splitting of the elastoplastic wave front: an elastic precursor is followed by a slower plastic loading wave. The peak in stress immediately behind the plastic wave is a result of a delay in the dislocation densities and velocities reaching the maximum values, and hence the rate of increase of tangential stress momentarily exceeds the decay. This behaviour has been observed also for idealised (non-work-hardening) rate-dependent relaxation models [23].

In the vicinity of the contact surface non-physical peaks in strain-hardening are observed as local increases in the equivalent stress. The origin of these artefacts is unequivocally attributable to entropy errors that manifest as a result of the numerical methods, and have been observed elsewhere $[1,33]$ for similar testcases (also known as overheating errors due to the consequential increase in temperature). Whilst we do not set out here to modify the numerical methods to eliminate the overheating problem, we shall provide evidence to support the argument that these are directly responsible for the observed anomaly in strain-hardening. We do this by reverting to modifying the equation set to include solution of the evolution of entropy associated with inelastic deformations Eq. (2.8). The entropy $\mathscr{S}^{p}$ is then used solely for the computation of the hardening terms in the relaxation time. In this way the relaxation time becomes a function of the various state parameters: $\tau=\tau\left(F_{i j}, \mathscr{S}_{,} \mathscr{S}^{p}\right)$. Solution of the resulting system leads to significant reductions of the artificial hardening (Fig. 4(a)). Comparison of $\mathscr{S}^{p}$ and the entropy obtained from the specific internal energy (Fig. 4(b)) reveals that the values differ only 


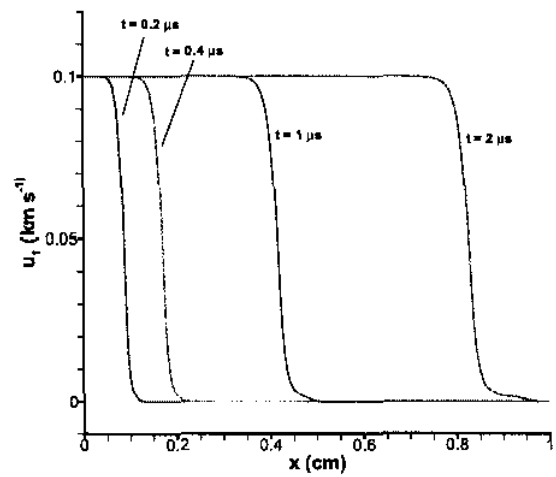

(a)

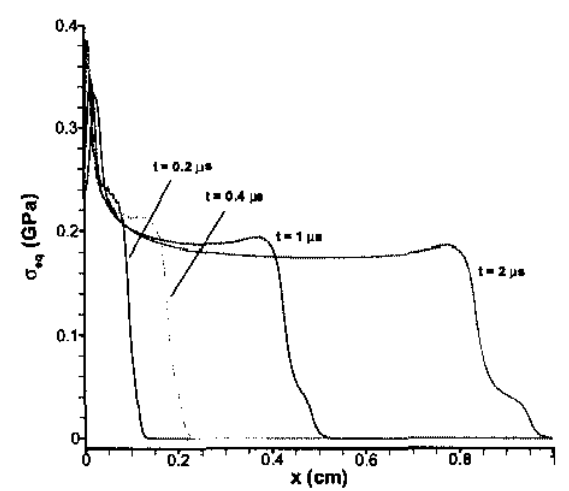

(b)

Figure 3: Profiles of (a) velocity and (b) equivalent stress for the one-dimensional impact testcase at the different indicated times.

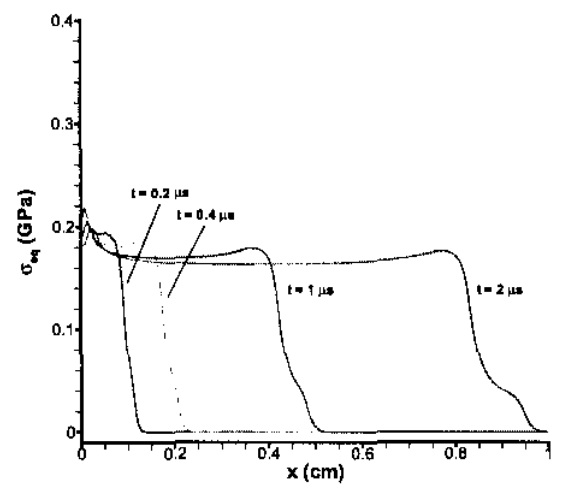

(a)

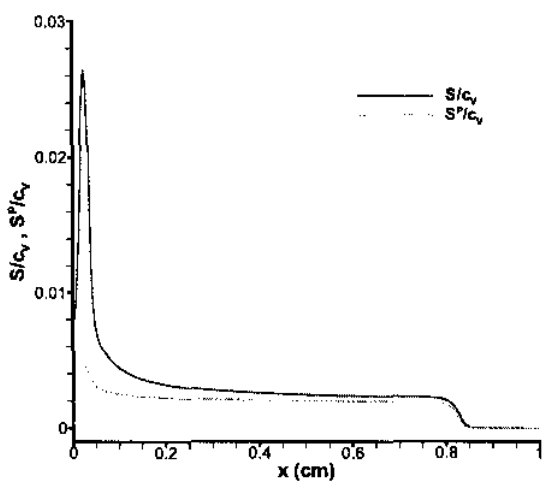

(b)

Figure 4: Profiles of (a) equivalent stress and (b) comparison of entropy computed from the internal energy and the plastic entropy for the one-dimensional impact testcase at the different indicated times.

marginally behind the shock and assures that. In the absence of overheating errors, it may then be adequate to use the total entropy as the measure of hardening in the relaxation model provided the shock contribution to total entropy is small in comparison to $\mathscr{S}^{p}$.

\subsection{Taylor bar impact studies}

In order to assess the performance of the proposed model for general multi-dimensional strains and outside the domain for which the relaxation time model is calibrated, cylinder 
Table 3: Comparison of simulated ratio of final stopping radii $r_{f}$ at the base of the cylinder to original radii $r_{0}$, and ratio of final length $L_{f}$ to original length $L_{0}$, with the experimental values reported in [35].

\begin{tabular}{||lllll||}
\hline $\begin{array}{l}\text { Impact velocity } \\
\left(\mathrm{m} \cdot \mathrm{s}^{-1}\right)\end{array}$ & \multicolumn{2}{c|}{ Experiment } & \multicolumn{2}{c||}{ Simulation } \\
\cline { 2 - 5 } & $L_{f} / L_{0}$ & $r_{f} / r_{0}$ & $L_{f} / L_{0}$ & $r_{f} / r_{0}$ \\
\hline \hline 130 & 0.770 & 1.30 & $0.795(+3)$ & $1.24(-5)$ \\
146 & 0.736 & 1.40 & $0.764(+4)$ & $1.33(-5)$ \\
$190^{\dagger}$ & 0.638 & 1.78 & $0.682(+7) / 0.675(+6)$ & $1.50(-16) / 1.59(-11)$ \\
\hline
\end{tabular}

${ }^{\dagger}$ Simulated values correspond to the grid sizes $20 \times 100 / 40 \times 200$.

$\mp$ Numbers in parenthesis indicate the percentage deviation from the experiment.

impact tests are considered. In these tests a finite sized cylindrical sample of the material is directed at high velocity on to a rigid (non-deformable) surface. If the stresses generated at the impact end of the sample exceed the elastic limit then material travelling into the plastic wave-front will deform inelastically, flowing radially outward and shortening the length of the bar. In modelling the event the degree of plastic dissipation governed by the relaxation model will thus influence the deceleration and hence final geometries at the point where all kinetic energy is diminished. If the material is too strong then the length of the bar will exceed what is observed experimentally, and vice-versa if the material is too weak, in this latter case accompanied also by excessive radial flow in the base region. Hence, it is widely recognised that these experiments provide a strenuous test of plasticity models since they feature strain, strain-rate and temperature fields the limits of which potentially exceeding those experienced in the uniaxial bar experiments used for model calibration. Deviations in computed and experimentally observed stopping geometries thus indicate specific inadequacies in the employed constitutive models.

Three different impact speeds are examined: $u_{2}=-130,-146,-190 \mathrm{~m} \cdot \mathrm{s}^{-1}$. The initial sample length and radius are $L_{0}=2.54 \mathrm{~cm}$ and $r_{0}=0.381 \mathrm{~cm}$ respectively. Unless mentioned otherwise, a computational mesh of $20 \times 100$ cells was used for all three tests. Computed values for the ratio of final stopping radii $r_{f}$ at the base of the cylinder to original radii $r_{0}$, and ratio of final length $L_{f}$ to original length $L_{0}$ for the selected speeds are presented in Table 3 along with deviations from the experimental values reported in [35]. The computational meshes and contours of non-dimensionalised entropy are shown in Fig. 5 for the terminal configuration. For the lower two impact speeds the values of final predicted stopping geometries are in good agreement with the experiment. The deviations of the simulated profiles are comparable to those reported in [35] for alternative, well-established plasticity models. At the highest impact velocity however, the deviations in the final base radii are quite large. It is in this region that the highest strains will be experienced. Hence, the values indicate that during the simulation the material is too hard when the strains are large. We of course expect some deviation from the experiment since the material constants for the model of relaxation time were determined using experimental data for copper taken from a source different from that for the experimental cylinder test results. Indeed variations in the test material will result in different material 


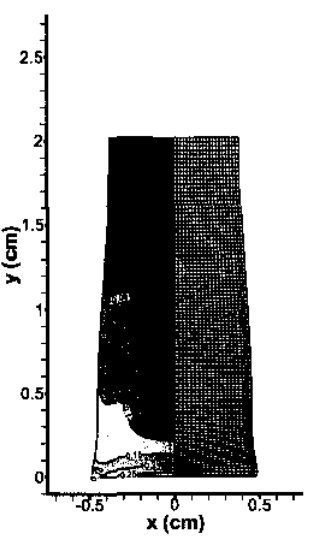

(a)

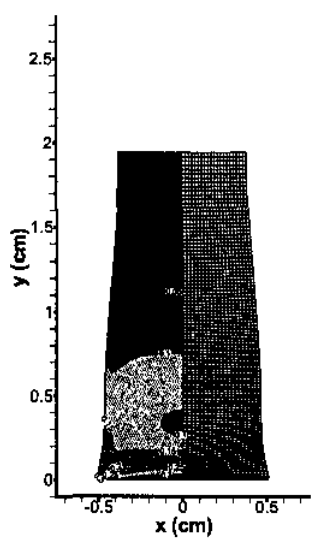

(b)

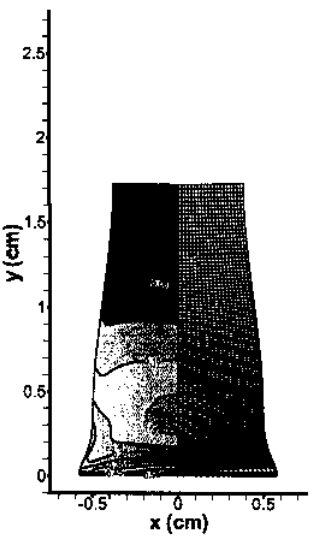

(c)

Figure 5: Computed results of the Taylor bar impact showing final stopping computational meshes and contours of $\mathscr{S} / C_{V}$ for the initial impact speeds (a) $u_{2}=-130 \mathrm{~m} \cdot \mathrm{s}^{-1}$; (b) $u_{2}=-146 \mathrm{~m} \cdot \mathrm{s}^{-1}$; (c) $u_{2}=-190 \mathrm{~m} \cdot \mathrm{s}^{-1}$.

parameters between samples. However, the predominant source of observed differences can be attributed to either or both similar entropy errors that were observed in the previous example, and/or the lack of a thermal softening mechanism in the relaxation model. Both of these deficiencies require further discussion.

It is known that decreasing the spatial discretisation error, when evaluating the governing differential equations, through increased grid resolution should act to reduce the volume of material affected by the entropy errors. Indeed it is seen that increasing the grid resolution leads to more accurate calculations of the final geometry, most significantly, as one would hope, in the base region (Fig. 6). As with the one-dimensional impact

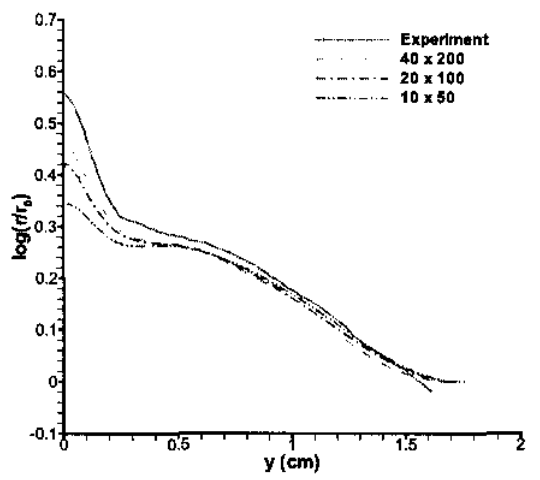

Figure 6: Computed final outer radius against $y=x_{2}$ for the different indicated grid resolutions in comparison with the experimental data extracted from [35]. 


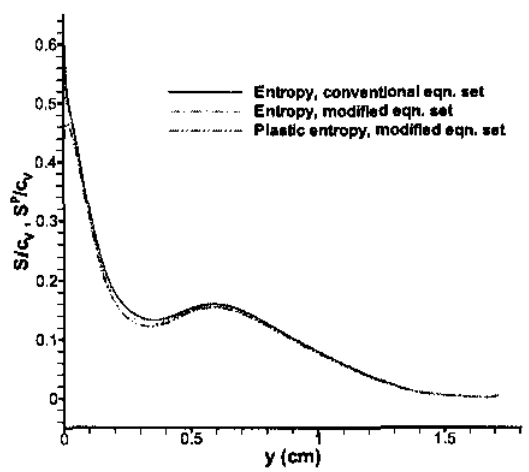

(a)

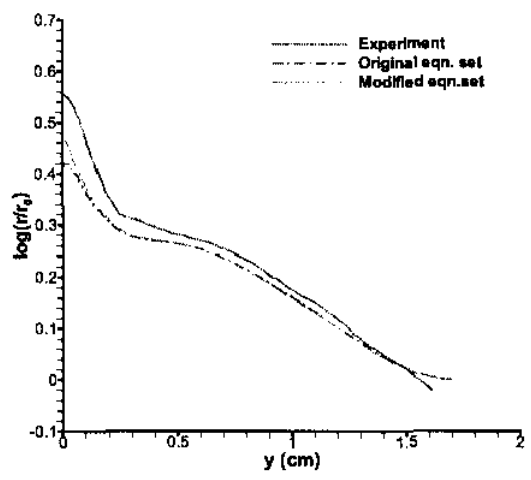

(b)

Figure 7: Final profiles of (a) computed outer radius against $y=x_{2}$ for the different indicated equation sets in comparison with the experimental data from [35], and (b) the total entropy and entropy associated with inelastic deformations along the $x_{2}$-axis.

example, the additional equation for entropy due to inelastic deformations alone Eq. (2.8) can be added in an attempt to overcome the entropy errors manifesting in the computed values of the relaxation time. By comparison it is seen that the artificial hardening is further reduced in this way (Fig. 7) and improved final geometries are achieved. Whilst the additional equation for entropy only alleviates the errors to a certain degree, the simulations serve to further strengthen the postulate that the observed decreased performance of the model is partially a result of entropy errors from the numerical scheme.

In respect to the lack of thermal softening mechanism, it is intuitive to observe the temperature distribution within the sample. Since the system is adiabatically isolated the peak values will be apparent in the terminal configuration. Taking the profile along the $x_{2}$-axis where we expect the highest temperatures to be experienced, it is found that the temperature rises by as much as $200 \mathrm{~K}$ (Fig. 8). At these elevated temperatures thermal

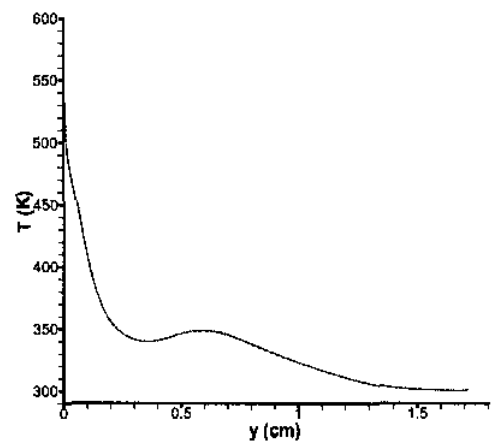

Figure 8: Final state of temperature along the $x_{2}$-axis. 
softening is quite profound and the flow stress should be reduced [16]. It is reasonable then to attribute the observed deviations in the final computed geometries primarily to the lack of thermal softening in the current model.

\section{Conclusions}

In this paper it has been shown how existing models for the viscoplastic deformation of isotropic materials can be reformulated to use plastic entropy as a parameter governing the onset of strain hardening. This is a novel approach not yet explored and has the potential to not only lead to a thermodynamically consistent formulation for material dynamics, but also eliminate the need for additional empirical constitutive equations for an irreversible history parameter. The crucial rate-independent relation between plastic strain and entropy turns out to be suitably simple to result in only limited additional complexity to the overall model when substituted into the formula for the relaxation time. The relaxation time was derived using established dislocation mechanics theory on the reasonable prospect that doing so would provide increased probability of success in application to regimes outside that for which the model was calibrated. It is mentioned, however, that the principle of substituting entropy for plastic strain could be used in conjunction with any existing empirical or otherwise model for strain-hardening materials provided the relation between the two state parameters is determined and the model is thermodynamically consistent. Consideration of the commonly employed empirical model of Ludwik for the flow stress of strain-hardening materials, which has been recognised to adequately describe many metals, hinted that the simple relationship that we determined is applicable in general. Of course, further analysis of a much larger range of materials is required to establish the validity of this proposition.

One-dimensional impact tests demonstrated the ability of the model to capture the characteristic elastoplastic wave structures. These also highlighted how use of total entropy to parametrise strain hardening, thus eliminating the solution of an additional evolution equation for plastic entropy and deemed an adequate approximation since entropy produced through the shock is comparably small, proved to be inadequate as a result of entropy errors in the vicinity of the contact surfaces inducing artificial hardening. Use of the additional equation for entropy produced solely through plastic work proved this mechanism to be the source of the observed errors. This elucidates the justification of development of improved numerical methods that do not suffer the artificial entropy production anomalies.

Cylinder impact tests demonstrated use of the model in a setting more akin to that which one would like to ultimately operate. A predominant motivating factor for choosing the cylinder impact test is to perform calculations using the proposed model where states are reached that are outside those for which the model was originally calibrated. Although good agreement is seen between computed and experimental final stopping geometric profiles, deformations in the base region are found to be overly restricted, such 
that the predicted base radius and length become increasingly inaccurate with increasing impact speed. One mechanism that has not been included within the proposed model of relaxation time is thermal softening. Under those conditions studied, regions of the sample will almost certainly experience elevated temperatures where thermal softening would manifest. This shortcoming of the model in it's current state can almost exclusively account for the loss of performance. It is nonetheless very much apparent that entropy can indeed parametrise work hardening in solid materials and warrants further work in this area.

\section{References}

[1] P. T. Barton, D. Drikakis, E. Romenski and V. A. Titarev, Exact and approximate solutions of Riemann problems in non-linear elasticity, J. Comp. Phys., 228 (2009) 7046.

[2] P. T. Barton, D. Drikakis and E. Romenski, An Eulerian finite-volume scheme for large elastoplastic deformations in solids, Int. J. Num. Meth. Eng., 81 (2010) 453.

[3] P. T. Barton and D. Drikakis, An Eulerian method for multi-component problems in non-linear elasticity with sliding interfaces, J. Comp. Phys., 229 (2010) 5518.

[4] C. Besaran and S. Nie, An irreversible thermodynamics theory for damage mechanics of solids, Int. J. Damage Mech., 13 (2004) 205.

[5] V. N. Dorovskii, A. M. Iskol'dskii and E. I. Romenskii, Dynamics of impulsive metal heating by a current and electrical explosion of conductors, J. Appl. Mech. Tech. Phys., 24 (1984) 454.

[6] N. Favrie, S.L. Gavrilyuk and R. Saurel, Solidfluid diffuse interface model in cases of extreme deformations, J. Comp. Phys., 228 (2009) 6037.

[7] R. P. Fedkiw, A. Marquina and B. Merriman, An isobaric fix for the overheating problem in multimaterial compressible flows, J. Comp. Phys., 148 (1999) 545.

[8] S. Gavrilyuk, N. Favrie and R. Saurel, Modelling wave dynamics of compressible elastic materials, J. Comp. Phys., 227 (2008) 2941.

[9] J. J. Gilman, Microdynamics of plastic flow at constant stress, J. Appl. Phys., 36 (1965) 2772.

[10] J. J. Gilman, Dislocation mobility in crystals, J. Appl. Phys., 36 (1965) 3195.

[11] P. P. Gillis and J. J. Gilman, Dynamical dislocation theory of crystal plasticity. I. The yield stress, J. Appl. Phys., 36 (1965) 3370.

[12] P. P. Gillis and J. J. Gilman, Dynamical dislocation theory of crystal plasticity. II. Easy glide and strain hardening, J. Appl. Phys., 36 (1965) 3380.

[13] J. J. Gilman, Dislocation dynamics and the response of materials to impact, Appl. Mech. Rev., $21(1968) 767$.

[14] S. K. Godunov, A. V. Zabrodin, M. Ya. Ivanov, A. N. Kraiko and G. P. Prokopov, Numerical Solution of Multidimensional Gas Dynamic Problems, Nauka, Moscow, 1976 (in Russian).

[15] S. K. Godunov and E. I. Romenskii, Elements of Continuum Mechanics and Conservation Laws, Kluwer Academic/Plenum Publishers, 2003.

[16] W. -G. Guo, Flow stress and constitutive model of OFHC Cu for large deformation, different temperatures and different strain rates, Baozha Yu Chongji/Explosion and Shock Waves, 25 (2005) 244 (in Chinese).

[17] D.J. Hill, D. Pullin, M. Ortiz and D. Meiron, An Eulerian hybrid WENO centered-difference solver for elasticplastic solids, J. Comp. Phys., 229 (2010) 9053.

[18] G. S. Jiang and C. W. Shu, Efficient implementation of weighted ENO schemes, J. Comp. Phys., 126 (1996) 202. 
[19] M. K. Keshavan and P. P. Gillis, On dislocation multiplication, J. Phys. F: Metal Phys., 5 (1975) 903.

[20] G. Kluth, B. Després, Discretization of hyperelasticity on unstructured mesh with a cellcentered Lagrangian scheme, J. Comp. Phys., 229 (2010) 9092.

[21] P. Ludwik, Elemente der Technologischen Mechanik, Julius Springer, 1909.

[22] L. A. Merzhievskii and S. A. Shamonin, Construction of the time dependence of the relaxation of tangential stresses on the state parameters of a medium, J. Appl. Mech. Tech. Phys., $21(1980) 716$.

[23] L. A. Merzhievskii and A. D. Resnyanskii, Shock-wave processes in metals, Combust. Explos., 20 (1984) 580.

[24] L. A. Merzhievskii and A. V. Paletsky, Calculations for diagrams of dynamic deformation of metals and alloys, Phys. Mesomechanics, 4 (2001) 79.

[25] G. H. Miller and P. Colella, A high-order Eulerian Godunov method for elastic-plastic flow in solids, J. Comp. Phys., 167 (2001) 131.

[26] G. H. Miller and P. Colella, A conservative three-dimensional Eulerian method for coupled solid-fluid shock capturing, J. Comp. Phys., 183 (2002) 26.

[27] J. A. Nelder and R. Mead, A simplex method for function minimization, Comp. J., 7 (1965) 308.

[28] E. Orowan, Problems of plastic gliding, Proc. Phys. Soc., 52 (1940) 8.

[29] K. Radhakrishnan and A. C. Hindmarsh, Description and use of LSODE, the Livermore solver for ordinary differential equations, LLNL report UCRL-ID-113855, 1993.

[30] E. I. Romenski, Thermodynamics and hyperbolic systems of balance laws in continuum mechanics, in: Godunov Methods: Theory and Applications, E. F. Toro (Ed.), Kluwer Academic/Plenum Publishers, 2001.

[31] H. L. Schreyer and P. J. Maudlin, Thermodynamically consistent relations involving plasticity, internal energy and thermal effects, Phil. Trans. R. Soc. A, 363 (2010) 2517.

[32] G. I. Taylor, The mechanism of plastic deformation of crystals, Proc. R. Soc. A, 145 (1934) 362.

[33] V. A. Titarev, E. Romenski and E. F. Toro, MUSTA-type upwind fluxes for non-linear elasticity, Int. J. Numer. Meth. Eng., 73 (2008) 897.

[34] Y. B. Zel'dovich and Y. P. Raizer, Physics of Shock Waves and High-Temperature Hydrodynamic Phenomena, v2, Academic Press, 1967.

[35] F. J. Zerilli and R. W. Armstrong, Dislocation-mechanics-based constitutive relations for material dynamics, J. Appl. Phys., 61 (1987) 1816. 